\title{
Postoperative myelopathy by undiagnosed cervical spine stenosis
}

\author{
Dong-Won Kim $\cdot$ Hyeong-Joong Yi $\cdot$ Kyu-Sun Choi
}

Received: 12 December 2014 / Accepted: 15 December 2014 / Published online: 24 January 2015

(C) Japanese Society of Anesthesiologists 2015

\section{To the Editor:}

An 82-year-old woman underwent laminectomy for spinal stenosis of L4-5 under a general anesthesia. Her past history and laboratory findings were unremarkable. She was obese and had moderate limitation of cervical range of motion. The endotracheal intubation was easily performed with the Pentax AWS ${ }^{\circledR}$ (Hoya Corporation, Tokyo, Japan). She was turned into the prone position in the usual manner. The operation lasted less than $2 \mathrm{~h}$, was uneventful. Blood pressure was stable during surgery. She felt an improvement of symptoms; however, fine finger motor difficulties and gait disturbance developed and became worse gradually over the next 3 days. Cervical-spine MRI showed a protruding intervertebral disc of C4-5, hypertrophy of ligament flavum, and spinal cord compression with increased signal intensity (ISI) on a sagittal T2-weighted image (Supplementary Fig. 1). An urgent C4-5 discectomy was performed. During the next 3 months, the patient's neurologic function gradually improved, although residual weakness remained.

Cervical spinal cord injury may occur after non-cervical spine surgery in patients with pre-existing cervical spondylosis [1]. It seems possible that vertebral artery ischemia due to intraoperative hypotension or vascular compromise related to head positioning, and hyperextension or

Electronic supplementary material The online version of this article (doi:10.1007/s00540-014-1966-8) contains supplementary material, which is available to authorized users.

\section{D.-W. Kim}

Department of Anesthesiology and Pain Medicine,

Hanyang University Medical Center, Seoul, Korea

\section{H.-J. Yi · K.-S. Choi $(\bowtie)$}

Department of Neurosurgery, Hanyang University Medical Center, 222 Wangsimniro, Seongdong-gu, Seoul 133-792, Korea e-mail: vertex-09@hanmail.net hyperflexion during endotracheal intubation and positioning might be contributable factors producing cervical spondylotic myelopathy (CSM) [2]. Although we tried to prevent excessive neck motion and unstable hemodynamic conditions in this case, postoperative spinal cord dysfunction occurred. Vascular compromise in just a mildly flexed cervical vertebra, precipitated by her pre-existing cervical spondylotic changes seems to be a plausible explanation of rapid progression of myelopathy.

Preoperative MRI of the cervical spine was not checked since initial subjective myelopathic symptoms and signs were negative. Practically, we are occasionally confronted with patients who have relatively mild or absent symptoms even though showing severe spinal cord compression with ISI on MRI. Even if preoperative MRI had shown ISI, she would be asymptomatic, and so an intraoperative event or posture might have had a role in producing spinal cord injury (SCI). If new ISI appeared on MRIs postoperatively, spinal ischemia or post-traumatic edema might have aggravated the SCI.

Based on these findings, clinicians should pay attention to the possibility of pre-existing cervical spondylosis before the procedure under general anesthesia for non-cervical spine disease in geriatric patients.

Conflict of interest None.

\section{References}

1. Hindman JB, Palecek JP, Posner KL, Traynelis VC, Lee LA, Sawin PD, Tredway TL, Todd MM, Domino KB. Cervical spinal cord, root, and bony spine injuries—a closed claims analysis. Anesthesiology. 2011;114:782-95.

2. Young IA, Burns SP, Little JW. Sudden onset of cervical spondylotic myelopathy during sleep: a case report. Arch Phys Med Rehabil. 2002;83:427-9. 